\title{
Article \\ Colonization of Clostridium butyricum in Rats and Its Effect on Intestinal Microbial Composition
}

\author{
Xianshu Luo, Qing Kong*(D), Yuming Wang, Xuefeng Duan, Peng Wang, Chenman Li and Yuchen Huan \\ School of Food Science and Engineering, Ocean University of China, Qingdao 266003, China; \\ 17864260858@163.com (X.L.); wangyuming@ouc.edu.cn (Y.W.); oucdxf@163.com (X.D.); \\ wangp960517@163.com (P.W.); dawnlcm@163.com (C.L.); huanyuchen79@outlook.com (Y.H.) \\ * Correspondence: kongqing@ouc.edu.cn
}

check for

updates

Citation: Luo, X.; Kong, Q.; Wang, Y.; Duan, X.; Wang, P.; Li, C.; Huan, Y. Colonization of Clostridium butyricum in Rats and Its Effect on Intestinal Microbial Composition.

Microorganisms 2021, 9, 1573. https:/ / doi.org/10.3390/microorganisms9081573

Received: 25 June 2021

Accepted: 19 July 2021

Published: 23 July 2021

Publisher's Note: MDPI stays neutral with regard to jurisdictional claims in published maps and institutional affiliations.

Copyright: (c) 2021 by the authors. Licensee MDPI, Basel, Switzerland. This article is an open access article distributed under the terms and conditions of the Creative Commons Attribution (CC BY) license (https:// creativecommons.org/licenses/by/ $4.0 /)$.

\begin{abstract}
Gut microorganisms participate in many physiological processes. In particular, Clostridium butyricum can modulate gut microorganisms and treat diseases. The colonization and persistence of strains in the gut contribute to beneficial effects, and the colonization by C. butyricum in the gut is currently unknown. We investigated the total intestinal contents of C. butyricum at $12 \mathrm{~h}, 24 \mathrm{~h}, 48 \mathrm{~h}$, and four and six days using real-time reverse transcription-PCR, after oral administration of C. butyricum to rats for seven consecutive days. We assessed the bacterial community structure using Illumina MiSeq sequencing. The results showed that $C$. butyricum was mainly colonized in the colon. The total content of $C$. butyricum in the gut increased significantly at $12 \mathrm{~h}$ after administration. Exogenous C. butyricum could still be detected in the gut six days after administration. Administration of $C$. butyricum significantly enhanced gut microbial diversity. The relative abundance of short-chain fatty acid-producing bacterial genera was shown to be higher than that of the control group, and treatment with C. butyricum elevated Firmicutes and diminished Bacteroidetes phyla compared with to the control group. These findings laid the foundation for the study of probiotic colonization capacity and the improvement of microflora for the prevention of gut diseases.
\end{abstract}

Keywords: Clostridium butyricum; real-time quantitative PCR; colonization; gut microorganisms

\section{Introduction}

The gut microbiome is an essential component of organisms, involved in many host physiological processes [1,2]. Indigenous bacteria in the mammalian gut have long been appreciated for their potential benefits to the host: provision of essential nutrients, metabolism of indigestible compounds, resistance to colonization by opportunistic pathogens, and contribution to the development of intestinal structure [3]. Healthy gut microbiota is essential for the overall health of humans and animals. The proper bacterial composition of the human microbiota prevents the development of disease, many metabolic diseases, inflammatory bowel diseases and even cardiovascular diseases have been reported to be associated with alterations in the microbial composition of the gut [4,5]. This homeostasis was achieved through interaction between the gut microbes and the host.

Clostridium is an anaerobic, Gram-positive, butyrate-producing bacillus. C. butyricum resides in the gastrointestinal tract and has a protective role against pathogenic bacteria and intestinal injury, via modulation of gut microbial metabolites [6], such as short-chain fatty acids (SCFAs) [7]. Duncan reported that C. butyricum could directly replenish butyrate as a butyrate-producing probiotic during repair of the damaged intestinal mucosa [8]. Obesity is associated with dysbiosis of intestinal flora, enhanced inflammation, poor barrier function, and reduced SCFA production, while butyrate-producing C. butyricum has been shown to treat the onset of high-fat diet-induced obesity and improve obesity-associated insulin resistance [9].

Probiotic-host interactions contribute to many physiological processes, including nutrient acquisition, development of the gut-specific immune system, and protection 
against infectious pathogens [2]. The World Health Organization defines probiotics as live microorganisms that, when administered in sufficient amounts, confer health benefits to hosts [10]. Successful colonization of the gut tract is a key factor in the ability of probiotics to exert sufficient host interactions to deliver health benefits. Although several studies have shown that the application of probiotics does not often lead to colonization, the survival of probiotics in the gut and their persistence in the host is a strong criterion for the successful effects of probiotics. The current literature shows that the probiotic bacteria Bacillus sp., Enterococcus sp., and Pediococcus sp. strain Ab1 can successfully colonize the digestive tract of different fish species, and P. acidilactici can remain in the intestine for at least 17 days without continual dietary administration [11]. However, some studies have also shown that probiotics ingested by humans are excreted in large quantities shortly after drug administration [12]. Similarly, Grimm et al. indicated that Bifidobacteria could not colonize the intestine of specific pathogen-free mice, but were able to colonize the intestine of germ-free mice [13]. An explanation for these results could be that competitive exclusion of species-native gut flora and individual differences among human subjects limit exogenous probiotic colonization. The colonization ability of different microorganisms varies, as does the colonization of the same microorganism in different individuals and sites of colonization. Many studies have reported beneficial effects of probiotic-host interactions on the host, and the native microorganisms in the host gut may have a long evolutionary history and have adaptive health properties to the host [14]. Therefore, it is reasonable to assume that local strains are better probiotic strains. As members of the autochthonous gut microbiota, it is likely that these species occupy specific niches that allow them to replicate and establish long-term stable populations [15].

C. butyricum is a native bacterium in the animal intestine, and the fate of $C$. butyricum in the intestine is a key bridge to study its interactions with the host. Studying the site of colonization and residence time of C. butyricum in the intestine is an essential part of exploring its effects on the host. There is a lack of research on the site of $C$. butyricum colonization and the effect of multiple gavage on the retention time of $C$. butyricum in the intestinal tract, except for Rumiko Sato [16], who studied the germination of C. butyricum in the intestine. Based on the above considerations, we established the first stable method (RT-qPCR) for detecting C. butyricum in the intestine and studied the colonization site of $C$. butyricum for the first time. Furthermore, we determined the retention time of exogenous C. butyricum in the intestine of specific pathogen-free Wistar rats for the first time and explored its effect on the microbial composition of the colon.

\section{Materials and Methods}

\subsection{C. butyricum Cultivation}

C. butyricum ZJUCB was preserved at the Ocean University of China. A thermostatic incubator (SHP-250, JINGHONG, Shanghai, China) was used to cultivate C. butyricum in Reinforced Clostridium Medium for $24 \mathrm{~h}$ at $37^{\circ} \mathrm{C}$. $16 \mathrm{~S}$ rDNA sequencing (27F: AGAGTTTGATCCTGGCTCAG, 1492R: TACGGCTACCTTGTTACGACTT) was performed on the broth of $C$. butyricum, and the sequencing results were compared on the NCBI website (https: / / www.ncbi.nlm.nih.gov; accessed on 25 December 2020) to confirm that the strain cultivated was $C$. butyricum. The $C$. butyricum fermentation broth was centrifuged at $10,000 \times g \mathrm{rpm}$ for $10 \mathrm{~min}$ and $20 \%$ trehalose was added to the bacterial pellets as a protective agent before freeze-drying. Freeze-dried bacteria were counted using the blood counting chamber, with a result of $10^{10} \mathrm{cfu} / \mathrm{mL}$. The freeze-dried powder was stored until usage.

\subsection{Primers and PCR Amplification}

Primer sets developed in this study were either from references or were designed based on the highly conserved regions of the genome using the GenBank database (https: / / www.ncbi.nlm.nih.gov/genbank, accessed on 19 July 2020) and using the DNAMAN 
for Windows, version 10. The primers were synthesized commercially by Ruibiotech. All primers used are in Table 1.

Table 1. Primer sets used to detect C. butyricum in rat intestine.

\begin{tabular}{|c|c|c|c|c|c|}
\hline & Primer Set & Primers Sequences $\left(5^{\prime}-3^{\prime}\right)$ & $\begin{array}{c}\text { Annealing Temp } \\
\left({ }^{\circ} \mathrm{C}\right)\end{array}$ & $\begin{array}{l}\text { Approximate } \\
\text { Amplicon Size } \\
\text { (bp) }\end{array}$ & $\begin{array}{l}\text { Reference or } \\
\text { Source }\end{array}$ \\
\hline \multirow{2}{*}{1} & Cbtu-1F & GTGCCGCCGCTAACGCATTAAGTAT & \multirow{2}{*}{72} & \multirow{2}{*}{213} & \multirow{2}{*}{ [17] } \\
\hline & Cbtu-1R & ACCATGCACCACCTGTCTTCCTGCC & & & \\
\hline \multirow{2}{*}{2} & Cbtu-2F & GCATCCAGCAGACTTAGCAG & \multirow{2}{*}{63} & \multirow{2}{*}{275} & \multirow{2}{*}{ This study } \\
\hline & Cbtu-2R & CTTCCGACTGTCTCATCTTC & & & \\
\hline \multirow{2}{*}{3} & Cbtu-3F & CAATGGTTGTGAATGCTGAGG & \multirow{2}{*}{60} & \multirow{2}{*}{142} & \multirow{2}{*}{ This study } \\
\hline & Cbtu-3R & CСAACTATGCTCATTTCGCCC & & & \\
\hline \multirow{2}{*}{4} & Cbtu- $4 \mathrm{~F}$ & CCTCAAATCCGCCTTCTGG & \multirow{2}{*}{58} & \multirow{2}{*}{102} & \multirow{2}{*}{ This study } \\
\hline & Cbtu-4R & GGATCTTGTTATCGTTCCG & & & \\
\hline \multirow{2}{*}{5} & Cbtu-5F & GAGTCTGATTCGGTTGTGGC & \multirow{2}{*}{60} & \multirow{2}{*}{135} & \multirow{2}{*}{ This study } \\
\hline & Cbtu-5R & ССТСТСТTССТTGAACTGGATG & & & \\
\hline
\end{tabular}

Each PCR mixture $(20 \mu \mathrm{L})$ contained $10 \mu \mathrm{L} 2 \times$ Phanta Max Buffer, dNTP Mix (10 mM each), $0.8 \mu \mathrm{L}$ Forward primer, $0.8 \mu \mathrm{L}$ Reverse primer, $1 \mu \mathrm{L}$ of bacterial template DNA, $0.4 \mu \mathrm{L}$ Phanta Max Super-Fidelity DNA Polymerase (Vazyme, Vazyme Biotech Co., Ltd., Nanjing, China), and $6.6 \mu \mathrm{L}$ nuclease-free water. Determining the optimal annealing temperature for each primer set (Table 1) and testing the specificity of each primer set was done by using Applied biosystems 2720 Thermal Cycler (Applied Biosystems, Thermo Fisher, Waltham, MA, USA). The PCR program consisted of 1 cycle with a DNA Pre- denaturation step at $95{ }^{\circ} \mathrm{C}$ for $30 \mathrm{~s}$ and 35 cycles with a DNA denaturation step at $95^{\circ} \mathrm{C}$ for $15 \mathrm{~s}$; followed by an annealing step for $15 \mathrm{~s}$ and elongation step at $72{ }^{\circ} \mathrm{C}$ for $45 \mathrm{~s}$. The PCR was completed with a final elongation step at $72{ }^{\circ} \mathrm{C}(5 \mathrm{~min})$.

\subsection{Real-Time qPCR}

Real-time quantitative PCR was performed on Thermo Lifetech ABI QuantStudio 3 from Applied Biosystems (Applied Biosystems, Thermo Fisher, America). All amplification reactions were carried out in 96-well optical-grade PCR plates in triplicate (Applied Biosystems, Thermo Fisher, America), each with $20 \mu \mathrm{L}$, sealed with optical sealing tape (Ruibiotech, Qingdao, China). Amplification reactions were done with $10 \mu \mathrm{L}$ SYBR ${ }^{\circledR}$ Green Realtime PCR Master Mix (QKD-201T, TOYOBO, Osaka, Japan), $0.8 \mu \mathrm{L}$ Forward primer, $0.8 \mu \mathrm{L}$ Reverse primer, $6.4 \mu \mathrm{L}$ nuclease-free, and $2 \mu \mathrm{L}$ of template DNA or nuclease-free (Primer sets was the same as PCR amplification). Controls without templates were included to ensure that no unspecific breakdown or amplification occurred. The amplification was done with the following temperature profiles: one cycle of $90{ }^{\circ} \mathrm{C}$ for $60 \mathrm{~s} ; 40$ cycles of $95^{\circ} \mathrm{C}$ for $15 \mathrm{~s}$ and Annealing temperature for $60 \mathrm{~s}$. Finally, melting curve analysis for assessing amplicon specificity. Quantitation was done by using standard curves made from known concentrations of pure culture $C$. butyricum containing the respective amplicon for each set of primers (Figure S1: Supplementary Materials). Efficiency was calculated from a linear regression curve through the data points, using the following equation: $\mathrm{E}=-1+10^{(-1 / \text { slope })}$. Primer set Cbtu-a showed satisfactory efficiency values ranging between 90 and 110\% with an $\mathrm{R}^{2}$ higher than 0.999 (Figure S1).

\subsection{Animals and Experimental Groups}

All animal procedures were performed by the guidelines of the ethical committee of experimental animal care at the College of Food Science and Engineering, Ocean University of China.

Male Wister rats weighing 280 to $300 \mathrm{~g}$ were purchased from Jinan Pengyue Experimental Animal Breeding Co., Ltd., Jinan, China. Thirty Wistar rats were randomly assigned to six groups according to their body weight $(n=5)$ : a control group (sterilized normal 
saline ( $\mathrm{NaCl}$ 0.8\%) was administered to the rats), and five test groups treated C. butyricum (C. butyricum freeze-dried powder dissolved in sterilized normal saline $\left(10^{8} \mathrm{CFU} / \mathrm{mL}\right)$ were administered to the rats). Test groups included: rats were sacrificed on day 6 (group A), on day 4 (group B), on day 2 (group C), on day 1 (group D), at 12 h (group E), after 7 consecutive days of gavage with $C$. butyricum. Rats were kept in individually ventilated cages in a pathogen-free animal facility at a temperature $\left(20-22{ }^{\circ} \mathrm{C}\right)$, humidity $(50-60 \%)$, and a pre-set light-dark cycle (12 h:12 h) was raised for 21 days. During the experimental periods, rats had free access to tap water and chow. Five animals were sacrificed for each observation at various time points (Table S1), and the entire intestinal tract, from the gullet to the rectum was removed. The contents of the cecum and the colon were completely flushed out with normal saline, and the contents were collected and stored at $-80{ }^{\circ} \mathrm{C}$ until usage.

\subsection{Genomic DNA Extraction from Pure Culture Strains and Fecal Samples of Rat Intestinal}

Both pure cultured bacterial genomic DNA and lyophilized bacterial genomic DNA were extracted by the Tiangen bacterial genomic DNA extraction kit (DP302-02, TIANGEN, Beijing, China). Genomic DNA was extracted from fecal samples using the Tiangen fecal genomic DNA extraction kit (DP328-02, TIANGEN, Beijing, China). Fresh fecal samples were collected from the cecum and colon of each mouse under aseptic conditions, and they were immediately immersed in liquid nitrogen and stored at $-80^{\circ} \mathrm{C}$ until subsequent analysis. The genomic DNA from a fecal sample of the cecum and colon was extracted by the Tiangen fecal genomic DNA extraction kit (DP328-02, TIANGEN, Beijing, China) according to the manufacturer's instructions. In all cases, the extracted DNA concentration was quantified by using a NanoDrop spectrophotometer ND 3.01000 (NanoDrop Technologies, Wilmington, DE, USA). The purity of the extracted DNA was checked by $1 \%$ agarose gel electrophoresis.

\section{6. $16 S$ rDNA V3-V4 Region Sequencing and Bioinformatics Analysis}

The V3 and V4 regions of the 16S rRNA gene were sequenced on an Illumina Miseq platform at Majorbio Bio-Pharm Technology Co. Ltd. (Majorbio, Shanghai, China). On the Uparse platform (http://drive5.com/uparse/, accessed on 25 December 2020), the sequences obtained were picked into operational taxonomic units (OUT) by clustering 97\% sequence similarity and classified at various taxonomic ranks (phylum, order, class, family, and genus) [18]. Alpha diversity and unweighted principal coordinate analysis plots using the phylogenic tree-based unifrac distance metric were generated using scripts from the QIIME package [19].

\subsection{Statistical Analysis}

Statistical analysis of the result of the six groups was performed using Students t-tests and a two-way ANOVA followed by Tukey's post-hoc test was used to determine the significance. All RT-qPCR data and sequencing data were analyzed using GraphPad Prism Version 8.0 and the online Majorbio-Sanger cloud platform (https:/ / cloud.majorbio.com, accessed on 25 December 2020).

The Illumina sequencing raw data were deposited in the NCBI sequence read archive database and assigned the accession no. PRJNA730420.

\section{Results}

\subsection{Specificity of Primer}

All primer sets used for PCR amplification of a single system of C. butyricum genomic DNA had high specificity, and only gave positive results for the corresponding target bacteria with the expected product size (Figure 1a). However, only one primer set, Cbtu1 , had high specificity in the complex PCR amplification system of intestinal microbial genomic DNA (Figure 1b,c). 
a

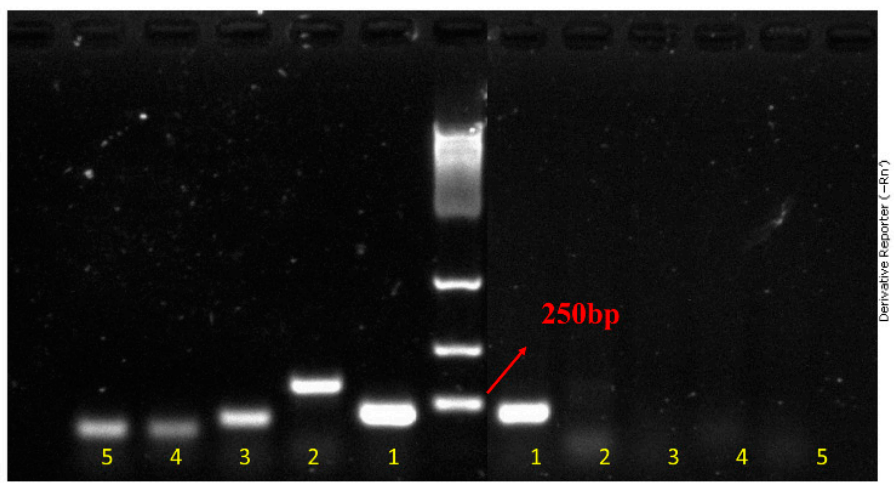

C

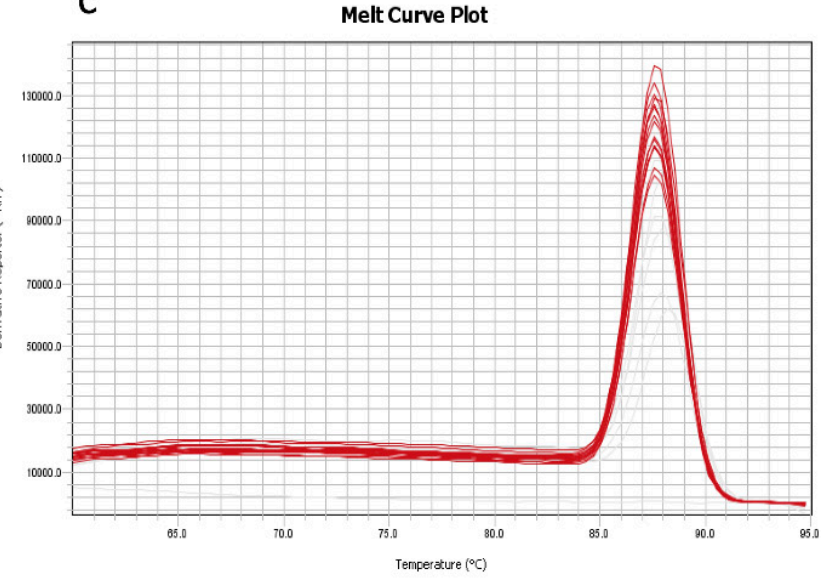

Figure 1. The primer set Cbtu-1 had high specificity. (a) All primer sets give positive results in a single system for PCR amplification (Primer sets 1, 2, 3, 4, and 5 are the same as Table 1). (b) Primer set Cbtu-1 amplifies the target product accurately in the complex system of intestinal microbial genomic DNA with a product length of $213 \mathrm{bp}$. (c) The melting curve plot of RT-qPCR amplification of primer set 1 is a single peak.

\subsection{Quantitation of C. Butyricum Populations in Rat Intestinal}

RT-qPCR was used to detect changes in the quantity of $C$. butyricum in the cecum and colon at $12 \mathrm{~h}, 24 \mathrm{~h}, 48 \mathrm{~h}$, and four and six days after oral administration of $C$. butyricum to rats for seven consecutive days and to determine colonization sites of endogenous $C$. butyricum in the intestine of specific pathogen-free Wistar rats. The results showed that the highest levels of $C$. butyricum were found in the cecum and colon at $12 \mathrm{~h}$ after seven consecutive days of $C$. butyricum administration. C. butyricum content was significantly increased in the cecum and colon at $24 \mathrm{~h}, 48 \mathrm{~h}$, and day 4 after administration compared to the control group administered sterile saline for seven consecutive days $(p<0.01)$ (Figure 2a). C. butyricum levels were lowest in the cecum of rats at six days after cessation of administration and increased in this group, but not significantly $(p>0.05)$ compared to the control group (Figure 2a). It was found that the content of $C$. butyricum in the colon was highest after administration of both sterile saline and exogenous $C$. butyricum by comparing C. butyricum in the cecum and colon (Figure 2b). In summary, both the cecum and colon, were the sites of colonization of endogenous $C$. butyricum, but the content in the colon was higher than that in the cecum. Second, the content of $C$. butyricum in the intestine was maintained at a higher level for at least six days after continuous administration of $C$. butyricum to rats.

\subsection{C. butyricum Changes the Composition of Rat Intestinal Flora}

The composition of the rat intestinal flora was studied via sequencing the $16 \mathrm{~S}$ bacterial genes. The unweighted UniFrac principal co-ordinate (PCoA) analysis showed that the composition of the intestinal flora of rats intragastrically administered with C. butyricum was different from that of the control group (Figure 3b). This was also evidenced by the differences between the experimental and control groups at the gate and genus levels (Figure $3 a, c)$. By assessing the diversity of $\alpha$, it was found that after administration of $C$. butyricum lyophilized powder for seven consecutive days in rats, there was a significant difference between the intestinal microbial richness and diversity $(p<0.01)$ (Figure 3d). 
a

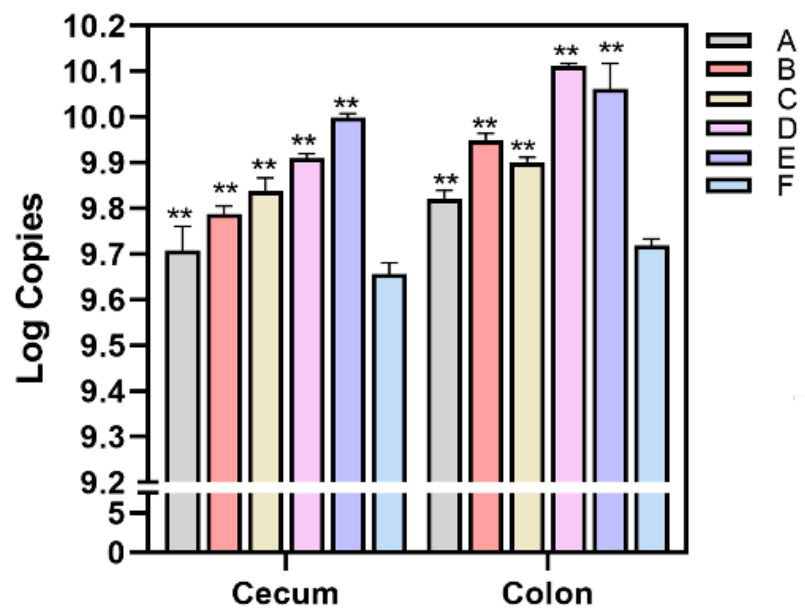

b

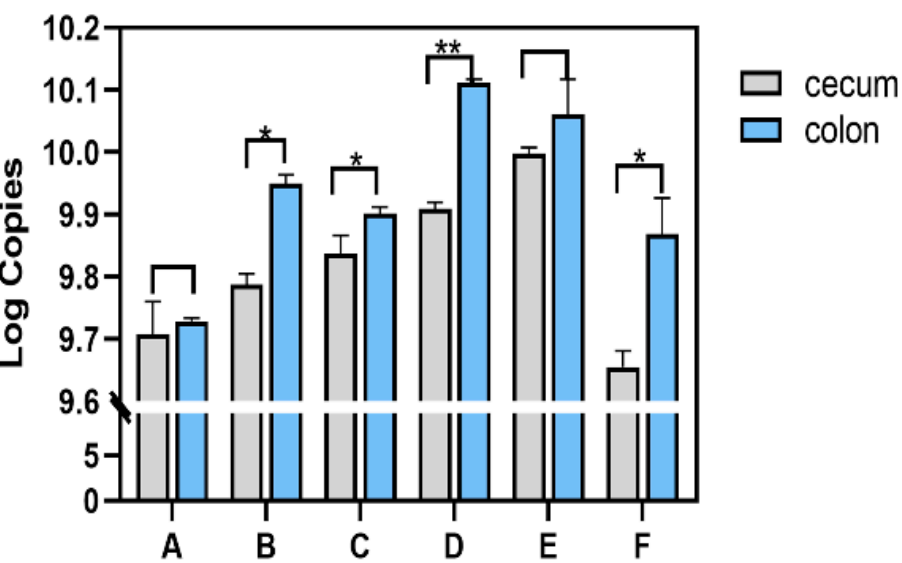

Figure 2. The level of Clostridium butyricum in the cecum and colon increased significantly after 7 consecutive days of gavage with C. butyricum. Raw data from RT-qPCR amplification were analyzed and plotted on GraphPad Prism Version 8.0 with two-way ANOVA to determine the significance. (a) Compared with group $\mathrm{F}$, there is a highly significant increase in the colon, C. butyricum in the cecum have no significant increase on day 6 (group A) after administration. (b) C. butyricum is more abundant in the colon than in the cecum. Results are shown as mean \pm SEM (n=3). ${ }^{*} p<0.05$ and ${ }^{* *} p<0.01$ compared to the control group (group F). A: Day 6th after administration; B: day 4th after administration; C: day 2nd after administration; D: $24 \mathrm{~h}$ after administration; E: $12 \mathrm{~h}$ after administration; F: administration of sterile saline group (control).

Firmicutes and Bacteroides were found to be the most abundant phyla in intestinal microbes (Figure 3a). When lyophilized C. butyricum powder was administered to rats for seven consecutive days, the ratio of Firmicutes and Bacteroides in the intestinal microbes of the experimental group changed significantly $(p<0.01)$. The changes in the ratio of Firmicutes/Bacteroides in group $\mathrm{E}$ (rat intestinal microbes at $12 \mathrm{~h}$ after gavage) were the largest, and Bacteroides increased by about two times compared to the control group (Group E: $40.47 \%$, Con group: 17.26\%) (Figure 3e). Interestingly, after observing the experimental data of groups A, B, C, and D, we found that after the administration of C. butyricum, with the extension of feeding time, the ratio of Firmicutes/Bacteroides was gradually recovered and, by day 6 , the content of Bacteroides and Firmicutes in rats had returned to normal levels (Figure $3 \mathrm{e}$ ).

We then evaluated the differences in the gut microbes of each group at the genus level in different periods and found that some genera showed significant changes $(p<0.01)$. First, the percentage of Lactobacillus in the intestinal microbes of the experimental group decreased by about six-fold compared to the control group (experimental group: 5.07\%; control group: $33.35 \%$ ) (Figure $4 \mathrm{~b}, \mathrm{f})$. Conversely, the content of other bacteria producing butyrate, acetate, and propionate increased. For example, the percentage of Alloprevotella increased from $4.70 \%$ to $8.51 \%$ (Figure 4c), the percentage of unclassified_Lachnospiriraceae increased from $4.19 \%$ to $8.52 \%$ and the percentage of norank_Muribaculaceae increased from $8.58 \%$ to $11.65 \%$ (Figure $4 \mathrm{a}, \mathrm{d}$ ). Second, we analyzed the composition of intestinal microbes at different time points after administration of $C$. butyricum and found that the relative abundance of unclassified_Lachnospiriraceae and Lachnospiriraceae_NK4A136_group showed an increasing trend within four days after administration of C. butyricum. By day 6 , the relative abundance began to decrease (Figure $4 \mathrm{~d}, \mathrm{e}$ ). However, norank_f muribaculaceae continued to increase on day 6 after administration, and the relative abundance of norank $f$ muribaculaceae was approximately twice that of the control group $(16.96 \%$ on the day 6$)$ (Figure 4a). Notably, an increase in the relative abundance of Allprevotella only appeared $12 \mathrm{~h}$ after gavage. After $24 \mathrm{~h}$, it returned to the normal level, and the relative abundance 
of Allprevotella remained at the normal level after $48 \mathrm{~h}$ and four and six days (Figure 4c). Overall, continuous gavage of $C$. butyricum changed the relative abundance of intestinal microbes in rats within a certain period, and the experimental results showed that some effects persisted.

a

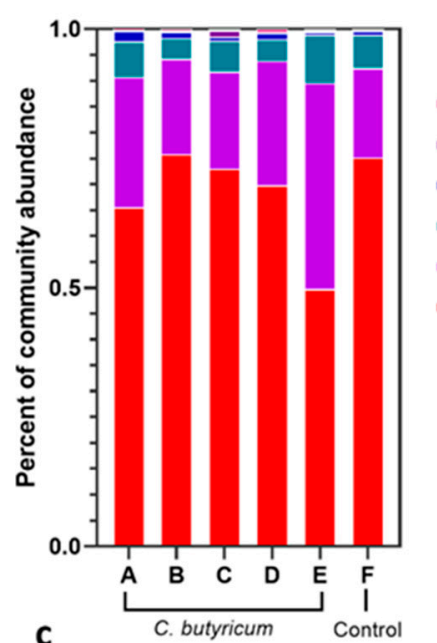

C
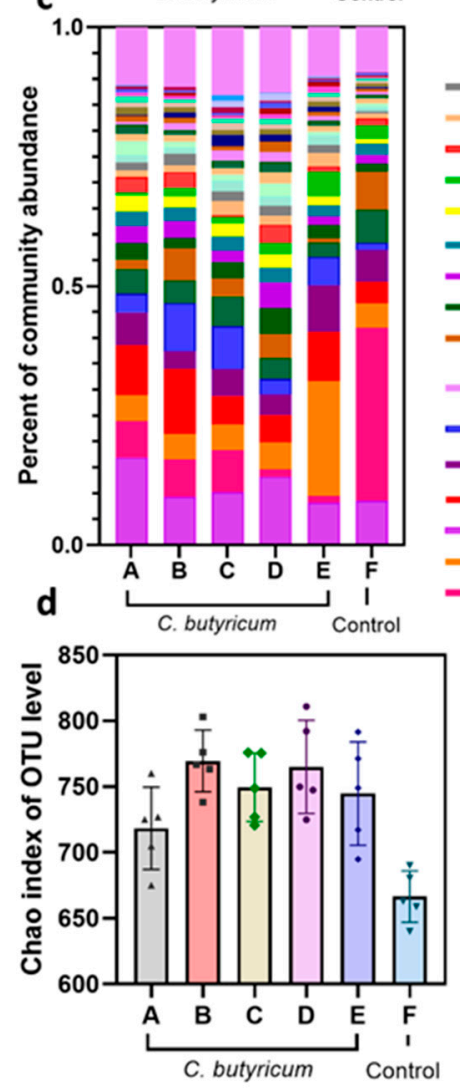

Phylum

- Others

- Proteobacteria

- Actinobacteriota

- Desulfobacterota

- Bacteroidota

- Firmicutes

Genus

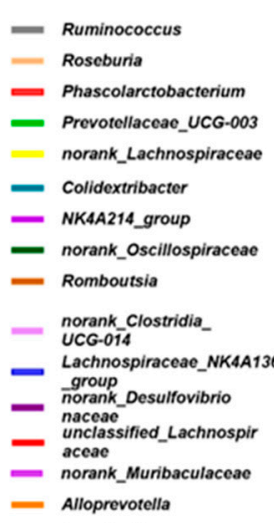

- Alloprevotella

- Lactobacillus

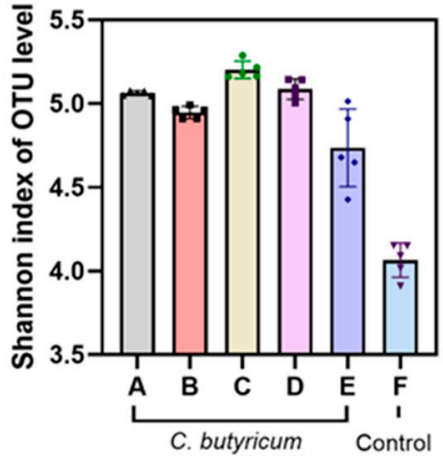

- others

- Anaerostipes

- UCG-003

- UCG-005

- Oscillibacter

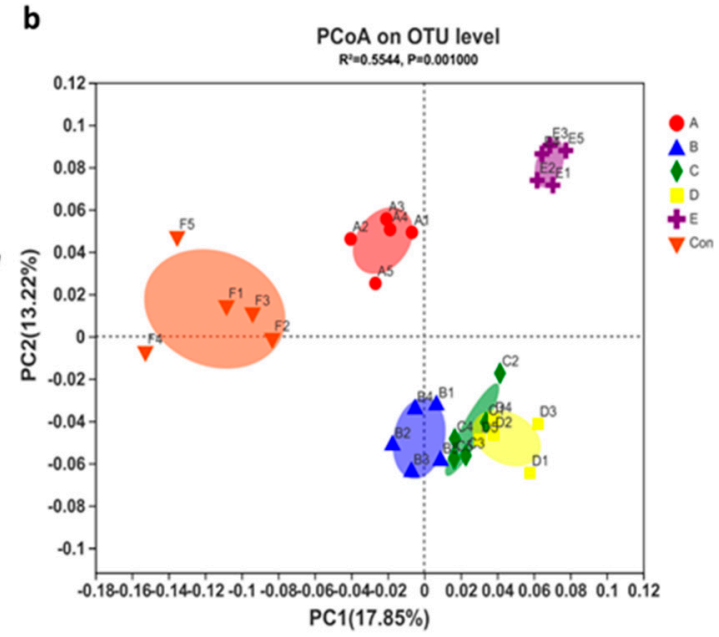

- Escherichia-Shigella

- Eubacterium_siraeum_group

- Christensenellaceae_R.7_group

- unclassified_Prevotellaceae

- Prevotellaceae_NK3B31_group

- norank_Ruminococcaceae

- norank_Peptococcaceae

- Eubacterium_xylanophilum_group

- norank_Eubacterium_coprostanoli

genes_group

- Oscillospiraceae

- Lachnospiraceac_UCG.006

e

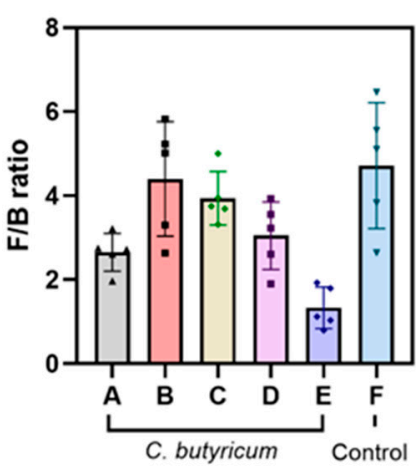

Figure 3. C. butyricum alters the intestinal flora of Wistar rats. Community abundance percentage plots showing the bacterial phyla and genera most differing in abundance between the treatment groups. (a,c) The colors of community abundance percentage plots represent the relative percentage of microbial families assigned to each sample. (b) Beta diversity of gut microbes analyzed by PCoA. PCoA used unweighted unifrac analysis to calculate the distances between the 6 groups in the colonic stool samples. The percentage shown on each axis explains the proportion of each dimension. (d) 16S rDNA V3-V4 sequencing to evaluate Chao richness and Shannon diversity. (e) The Firmicutes / Bacteroidetes ratio was calculated as a biomarker of intestinal flora dysbiosis. A: day 6th after administration; B: day 4th after administration; C: day 2nd after administration; D: $24 \mathrm{~h}$ after administration; E: $12 \mathrm{~h}$ after administration; F: administration of sterile saline group (control). 
a

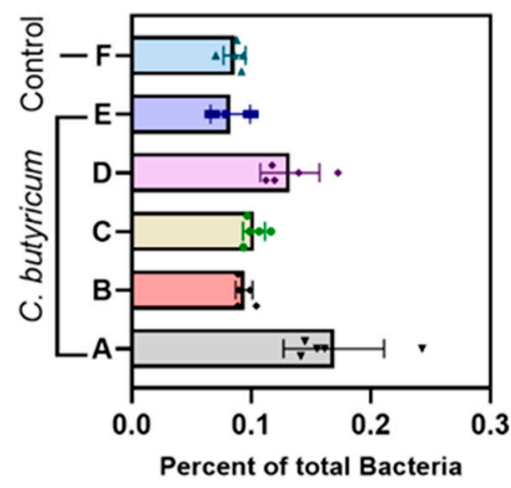

d unclassified_Lachnospiraceae

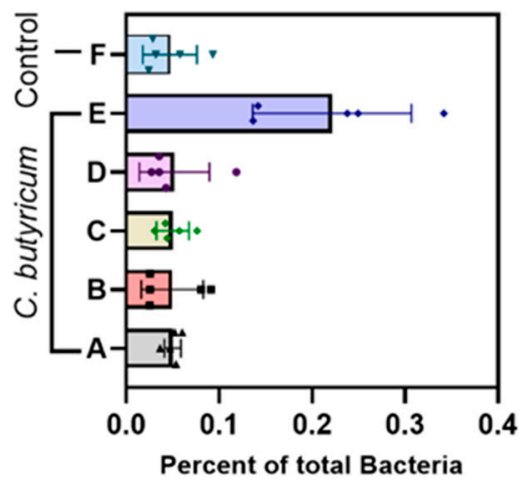

b
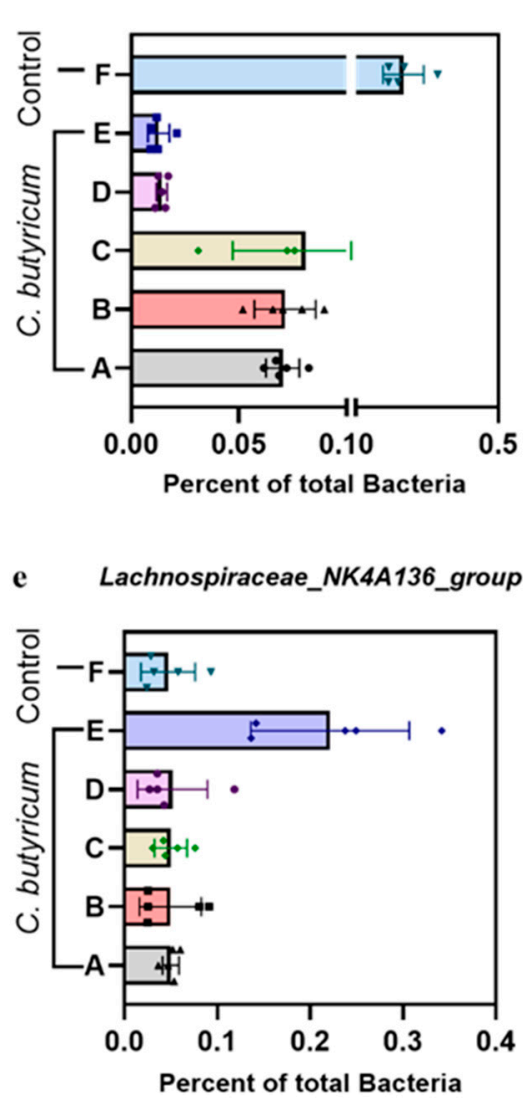

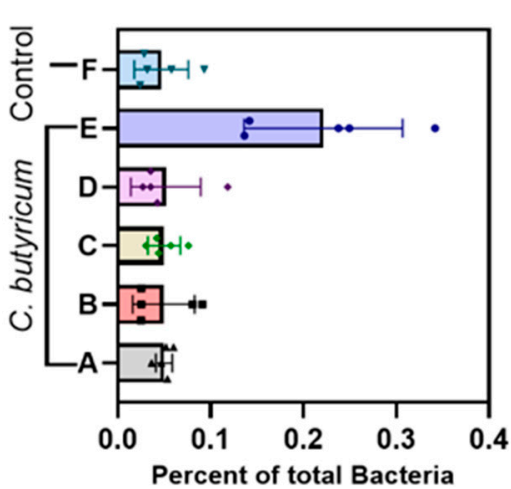

f

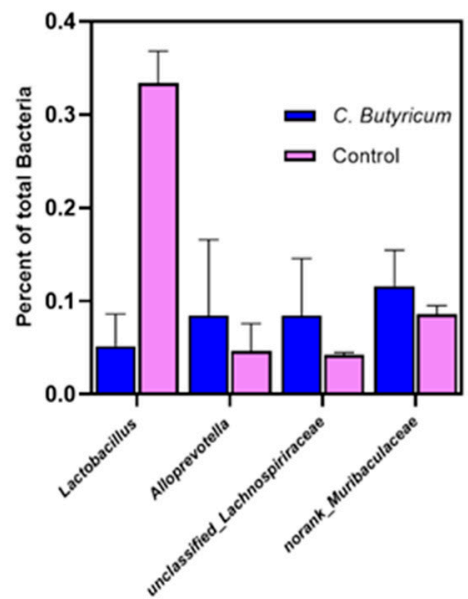

Figure 4. Histogram of the percentage of bacterial genera showing the differences in the abundance of the top five bacterial genera between treatment groups. (a-e) The genera norank_Muribaculaceae, Lactobacillus, Alloprevotella, unclassified_Lachnospiraceae, and Lachnospiraceae_NK4A136_group changed significantly after gavage of C. butyricum. (f) Changes in the relative abundance of the genera norank_Muribaculaceae,_Lactobacillus, Alloprevotella, unclassified_Lachnospiraceae, and Lachnospiraceae_NK4A136_group in the administered group compared to the control group. A: day 6th after administration; B: day 4th after administration; C: day 2nd after administration; D: $24 \mathrm{~h}$ after administration; E: $12 \mathrm{~h}$ after administration; F: administration of sterile saline group (control).

\section{Discussion}

Intestinal flora is altered in many diseases [20]. For example, inflammatory bowel disease, hypertension, obesity, and diabetes are closely related to intestinal flora imbalance [21-24]. Probiotics are an effective way to regulate intestinal flora imbalance and can be used as an effective treatment for many diseases. C. butyricum exists in the intestine and has many beneficial functions [25-27]. This study aimed to provide some further evidence for the possibility that $C$. butyricum can be used as a probiotic, by clarifying the site of colonization, the persistence of exogenous C. butyricum, and its effect on microbial composition in the intestine.

We established a method to detect the content of C. butyricum in the intestinal tract, showing the relative abundance of $C$. butyricum in the cecum and colon after oral administration of C. butyricum to rats for seven consecutive days and the distribution of endogenous C. butyricum in the intestine. In addition, we have expanded the existing knowledge on the regulation of the richness and diversity of gut microbes. C. butyricum protects the intestinal 
flora of colon and rectal cancers [28]. Kanai et al. and Seo et al. have shown that the presence of $C$. butyricum in the intestine inhibits pathogenic bacteria and protects against intestinal injury $[6,28]$. To carry out their functional activities, C. butyricum must be able to survive in the gastrointestinal tract and persist, at least transiently, in the host. Bacillus subtilis spores can germinate and even transiently colonize the intestine. However, the spores themselves might also exert an immunostimulatory effect to exclude colonization of the intestine by harmful pathogens [29]. This suggested that using exogenous C. butyricum by administering it to specific pathogen-free Wistar rats to extend the probiotic potential of C. butyricum in this study was theoretically valid. A previous study by Rumiko Sato [16] showed that $C$. butyricum spores tolerated gastric acid but did not germinate in the front of the stomach and small intestine due to low $\mathrm{pH}$ and high oxidation-reduction potential. C. butyricum spores germinated in the proximal and middle small intestine, cecum, and colon; at the same time, the oxidation-reduction potential in the intestine was immediately reduced after the spores germinated [30]. The findings in this study showed that after administration of exogenous C. butyricum lyophilized powder for seven consecutive days, the content of $C$. butyricum in the cecum and colon of rats increased significantly $(p<0.01)$ compared to the control group. Administration of sterile saline in the control group showed that the colon was the colonization site of endogenous C. butyricum. Furthermore, although the content of $C$. butyricum in the cecum and colon decreased with increasing feeding time, there was still a significant increase in the content of $C$. butyricum in the colon compared to the control group at day 6 after administration $(p<0.01)$. Further, Yue et al. [31] studied Lactobacillus casei SY13 short-term oral administration (once in one day) and long-term treatment (once daily, 27 times) on Lactobacillus casei SY13 colonization in the intestinal tract. The results showed that with short-term gavage, Lactobacillus casei SY13 cells remained in the intestine for less than three days, which is consistent with the results of the current research. However, for long-term gavage, the cells were retained in the intestine for seven days. Long-term oral administration prolonged the retention time of this bacterium. In this study, to analyze the effect of multiple administrations of C. butyricum on the level of colonization in the gut, we administered it for seven consecutive days. We concluded that multiple administrations enhanced the ability of C. butyricum to colonize the intestinal tract. After multiple administrations of C. butyricum to rats, although some C. butyricum spores were excreted by intestinal motility before germination, some C. butyricum spores germinated and grew. A possible explanation for the difference in results was that when microorganisms were supplemented for a long time, they could colonize the intestine because their reproduction rate was higher than the expulsion rate. However, the possibility that the results of this study show temporal persistence could not be excluded. It was reported that Bifidobacteria could not stably colonize in the intestine of specific pathogen-free mice but were able to colonize the intestine of germ-free mice and the highest bacterial concentration in the colonic lumen [13]. The competitive exclusion of the intestinal native flora and the differences between individual subjects limit the colonization of exogenous probiotics, which could explain the phenomenon of time-dependent colonization of the intestine by exogenous probiotics. Conversely, many researchers believe that microorganisms of human origin are important criteria for the selection of probiotics [14,32]. Although most probiotic strains have been derived from human feces or intestines, they are gradually excreted from the body after administration is stopped or shortly afterward. Most lactobacilli found in the mammalian gut are not the true intestinal inhabitants; they are retained because they meet the nutrient requirements of the organism. The colon has the thickest mucus layer, and butyrate affects the composition of the mucus layer [33]. Intestinal bacteria have adapted to colonize the mucus layer by adhering to intestinal mucus components, using mucus-derived nutrients, and sensing chemical cues for adaptation [34]. Although no specific groups of mucus-adhering bacteria have been identified, there is evidence that bacteria can bind directly to mucins by expressing specific proteins, pili, fimbriae, and flagella [35]. Therefore, probiotics remain mainly in the colon. In conclusion, after supplementation with exogenous $C$. butyricum for seven consecutive days, the levels of $C$. 
butyricum in the cecum and colon were significantly higher than those in the control group due to a greater colonization rate than expulsion rate of $C$. butyricum in the intestine; $C$. butyricum was retained in the colon for at least six days.

Intestinal bacteria are a key factor in regulating the digestion in the gastrointestinal tract and have a key immune effect on the colonization of pathogenic bacteria. These microorganisms prevent bacterial invasion by maintaining the integrity of the intestinal epithelial cells [36]. The main phyla in the gut microbes are Firmicutes, Bacteroidetes, Actinobacteria, Proteobacteria, Fusobacteria, and Verrucomicrobia. Firmicutes and Bacteroides account for $90 \%$ of the intestinal flora [36,37]. To clarify the impact of $C$. butyricum colonization on the composition of intestinal microbes, we performed 16S rDNA gene sequencing. Bioinformatics analysis showed that the composition of gut microbes was altered by C. butyricum. C. butyricum elevated Firmicutes and diminished Bacteroidetes phyla, compared to the control group. Lactobacillus in the gut showed a significant decrease after administration compared to the control group, which explains why Firmicutes showed a significant decrease after $C$. butyricum supplementation. Here, we also observed that the ratio of Firmicutes/Bacteroidetes decreased at $12 \mathrm{~h}$ after administration, but at $24 \mathrm{~h}$, the abundance of Firmicutes and Bacteroides gradually recovered. At day 6, the abundances of Firmicutes and Bacteroides returned to normal levels, but their proportions still showed an increase compared to the control group. The ratio of Firmicutes to Bacteroides is a sign of gut health. As previously shown, hypertension, obesity, and inflammatory bowel disease are associated with an increased ratio of Firmicutes/Bacteroides [25,26,28,38]. After administration, the intestinal flora gradually recovered because healthy rats had a complete composition of gut microbes, and undisturbed intestinal immune function played an important role. We also found that C. butyricum increased the abundance of SCFA-producing bacteria, such as unclassified_f Lachnospiriraceae, Lachnospiriraceae_NK4A136_group, Alloprevotella, and norank $f$ muribaculaceae. The composition of the intestinal microbiota differs at different times after administration. The relative abundance of colonic Alloprevotella, Lachnospiraceae_NK4A136_group and unclassified_Lachnospiraceae increased and reached its highest level at $12 \mathrm{~h}$ after administration. While norank_muribaculaceae continued to increase on day 6 after intragastric administration, their relative abundance was highest on day 6 . Hou et al. reported that norank_f muribaculaceae and Alloprevotella are negatively associated with obesity $[18,39]$. Zhang et al. pointed out that the main metabolites of Lachnospiriraceae is butyrate [40]. The abundance of the genera Alloprevotella and Lachnospiraceae_NK4A136_group abundance has been shown to be negatively correlated with inflammation [41]. Some studies have also proposed that increased Alloprevotella, Lachnospiraceae_NK4A136_group abundance, and fecal SCFA content are linked to a decrease in TLR4 signaling and LPS levels [42,43]. SCFAs, including acetate, propionate, butyrate, isobutyrate, and valerate, are important metabolites and information molecules in the intestinal flora. They are produced within the intestinal lumen by bacterial fermentation of mainly undigested dietary carbohydrates. In the cecum and large intestine, $95 \%$ of the produced SCFAs are rapidly absorbed by the colonocytes, while the remaining $5 \%$ are secreted in the feces $[44,45]$. SCFAs are the main energy source for epithelial cells and have many beneficial effects, such as improving barrier function and reducing mucosal inflammation [46]. In general, we believe that $C$. butyricum can play a beneficial role through its metabolites (mainly SCFAs) or by increasing the abundance of other SCFAproducing bacteria. After supplementation with exogenous $C$. butyricum, different genera of SCFA-producing bacteria in the intestine were increased at different times, with some showing short-term changes rapidly increasing after administration, while others increased in abundance some time after administration. Despite the differences in the time of appearance, the effects on intestinal microorganisms were definite. This result suggested that supplementation with exogenous C. butyricum promoted an increase in the relative abundance of SCFA-producing bacteria in the intestine, prolonging their beneficial effects on the host. 


\section{Conclusions}

Exogenous bacteria have difficulties colonizing the intestine because of competitive exclusion and individual differences. C. butyricum, a native intestinal bacterium, has the advantage of occupying specific niches in the intestine, but colonization is still difficult. In this study, we attempted to increase the level of $C$. butyricum colonization in the intestine and prolong the retention time of exogenous probiotics in the intestine by regulating the proliferation and expulsion rates. These results were reported by us: (1) C. butyricum is a native microorganism in the intestines and mainly colonizes the colon; (2) after gavage of C. butyricum to rats for seven consecutive days, it was retained in the intestinal tract for at least six days; and (3) C. butyricum changed the composition of the gut microbes.

This research suggested that $C$. butyricum is an intestinal native bacterium and that long-term intragastric administration facilitates colonization of $C$. butyricum in the colon. After seven consecutive days of exogenous C. butyricum supplementation, temporal colonization levels were observed during and at least six days after dietary supplementation. The residence of exogenous $C$. butyricum in the intestine altered the F/B ratio, a marker of intestinal health. In addition, the abundance of SCFAs (acetate, butyrate, and propionate)producing bacteria in the colon increased. However, a significant decrease in the abundance of Lactobacillus was observed. A shortcoming of our study was that after seven consecutive days of feeding exogenous C. butyricum, the feeding time was only six days. Based on this, we could not conclude with absolute certainty that $C$. butyricum colonized the colon. However, we believe that continuous supplementation with exogenous $C$. butyricum could prolong its residence time in the intestine. The presence of probiotics in the intestine might stimulate the beneficial effect of probiotics, but it has not been reported in the literature that probiotics need to colonize to exert health benefits on the host. This study prolonged the retention time of $C$. butyricum in the gut by adjusting the ratio of multiplication rate to expulsion rate and temporal colonization of C. butyricum in the gut. This would be a breakthrough point for the improvement of the health benefits of C. butyricum. As suggested in the study, the persistence of exogenous C. butyricum in the gut and the alteration in healthy gut microbes may be an entry point to study the role of C. butyricum in the prevention of certain diseases. Future studies should explore the impact of $C$. butyricum on the control of metabolic diseases associated with SCFAs.

Supplementary Materials: The following are available online at https:/ /www.mdpi.com/article/10 .3390 /microorganisms9081573/s1, Table S1: Administration time, execution time, and gavage dose for each group of rats. Figure S1: Amplification results of RT-qPCR, including amplification plot and melt curve plot of the standard curve.

Author Contributions: Conceptualization, Q.K. and X.L.; methodology, Q.K., X.L. and Y.W.; formal analysis, Q.K., X.D. and X.L.; investigation, X.L., X.D., P.W., C.L., Y.H. and Y.W.; writing-original draft preparation, Q.K. and X.L.; writing-review and editing. All authors have read and agreed to the published version of the manuscript.

Funding: National Natural Science Foundation of China (32072328), and the National Key R\&D Program of China (2019YFD0901705).

Institutional Review Board Statement: The study was conducted according to the guidelines of the ethical committee of experimental animal care at the College of Food Science and Engineering, Ocean University of China, and approved by the Animal Ethics Committee of Ocean University of China (Approved protocol no: SPXY2017050402, 10 October 2020).

Informed Consent Statement: Not applicable.

Data Availability Statement: The data that support the figures within this paper and other findings of this study are available from the corresponding author upon reasonable request.

Acknowledgments: We thank the National Natural Science Foundation of China (32072328) and the National Key R\&D Program of China (2019YFD0901705) for supporting this research.

Conflicts of Interest: The authors declare no conflict of interest. 


\section{References}

1. Engevik, M.A.; Versalovic, J. Biochemical features of beneficial microbes: Foundations for therapeutic microbiology. Bugs Drugs 2018, 5, 1-47.

2. Liu, M.; Xie, W.; Wan, X.; Deng, T. Clostridium butyricum modulates gut microbiota and reduces colitis associated colon cancer in mice. Int. Immunopharmacol. 2020, 88, 106862. [CrossRef]

3. Hooper, L.V.; Falk, P.G.; Gordon, J.I. Analyzing the molecular foundations of commensalism in the mouse intestine. Curr. Opin. Microbiol. 2000, 3, 79-85. [CrossRef]

4. Guarner, F.; Malagelada, J.-R. Gut flora in health and disease. Lancet 2003, 361, 512-519. [CrossRef]

5. Tremaroli, V.; Bäckhed, F. Functional interactions between the gut microbiota and host metabolism. Nature 2012, 489, 242-249. [CrossRef]

6. Seo, M.; Inoue, I.; Tanaka, M.; Matsuda, N.; Nakano, T.; Awata, T.; Katayama, S.; Alpers, D.H.; Komoda, T. Clostridium butyricum MIYAIRI 588 improves high-fat diet-induced non-alcoholic fatty liver disease in rats. Digest. Dis. Sci. 2013, 58, 3534-3544. [CrossRef]

7. Zhang, J.; Chen, X.; Liu, P.; Zhao, J.; Sun, J.; Guan, W.; Johnston, L.J.; Levesque, C.L.; Fan, P.; He, T.; et al. Dietary Clostridium butyricum Induces a phased shift in fecal microbiota structure and increases the acetic acid-producing bacteria in a weaned piglet model. J. Agric. Food Chem. 2018, 66, 5157-5166. [CrossRef] [PubMed]

8. Duncan, S.H.; Hold, G.L.; Barcenilla, A.; Stewart, C.S.; Flint, H.J. Roseburia intestinalis sp. nov., a novel saccharolytic, butyrateproducing bacterium from human faeces. Int. J. Syst. Evol. Microbiol. 2002, 52, 1615-1620. [CrossRef]

9. Shang, H.; Sun, J.; Chen, Y.Q. Clostridium butyricum CGMCC0313.1 modulates lipid profile, insulin resistance and colon homeostasis in obese mice. PLoS ONE 2016, 11, e0154373. [CrossRef] [PubMed]

10. de Vries, M.C.; Vaughan, E.E.; Kleerebezem, M.; de Vos, W.M. Lactobacillus plantarum survival, functional and potential probiotic properties in the human intestinal tract. Int. Dairy J. 2006, 16, 1018-1028. [CrossRef]

11. Li, X.; Ringø, E.; Hoseinifar, S.H.; Lauzon, H.L.; Birkbeck, H.; Yang, D. The adherence and colonization of microorganisms in fish gastrointestinal tract. Rev. Aquacult. 2019, 11, 603-618. [CrossRef]

12. Han, S.; Lu, Y.; Xie, J.; Fei, Y.; Zheng, G.; Wang, Z.; Liu, J.; Lv, L.; Ling, Z.; Berglund, B.; et al. Probiotic gastrointestinal transit and colonization after oral administration: A long journey. Front. Cell. Infect. Microbiol. 2021, 11. [CrossRef]

13. Grimm, V.; Radulovic, K.; Riedel, C.U. Colonization of C57BL/6 Mice by a potential probiotic Bifidobacterium bifidum strain under germ-free and specific pathogen-free conditions and during experimental colitis. PLoS ONE 2015, 10, e0139935. [CrossRef] [PubMed]

14. Walter, J. Ecological role of Lactobacilli in the gastrointestinal tract: Implications for fundamental and biomedical research. Appl. Environ. Microbiol. 2008, 74, 4985-4996. [CrossRef]

15. Frese, S.A.; Hutkins, R.W.; Walter, J. Comparison of the colonization ability of autochthonous and allochthonous strains of Lactobacilli in the human gastrointestinal Tract. Adv. Microbiol. 2012, 2, 11. [CrossRef]

16. Sato, R.; Tanaka, M. Intestinal distribution and intraluminal localization of orally administered Clostridium butyricum in rats. Microbiol. Immunol. 1997, 41, 665-671. [CrossRef]

17. Bartosch, S.; Fite, A.; Macfarlane, G.T.; McMurdo, M.E. Characterization of bacterial communities in feces from healthy elderly volunteers and hospitalized elderly patients by using real-time PCR and effects of antibiotic treatment on the fecal microbiota. Appl. Environ. Microbiol. 2004, 70, 3575-3581. [CrossRef] [PubMed]

18. Hou, D.; Zhao, Q.; Yousaf, L.; Khan, J.; Xue, Y.; Shen, Q. Consumption of mung bean (Vigna radiata L.) attenuates obesity, ameliorates lipid metabolic disorders and modifies the gut microbiota composition in mice fed a high-fat diet. J. Funct. Foods 2019, 64, 103687. [CrossRef]

19. Robles-Vera, I.; Toral, M.; de la Visitación, N.; Sánchez, M.; Gómez-Guzmán, M.; Romero, M.; Yang, T.; Izquierdo-Garcia, J.L.; Jiménez, R.; Ruiz-Cabello, J.; et al. Probiotics prevent dysbiosis and the rise in blood pressure in genetic hypertension: Role of short-chain fatty acids. Mol. Nutr. Food Res. 2020, 64, e1900616. [CrossRef]

20. Zhang, Y.-J.; Li, S.; Gan, R.-Y.; Zhou, T.; Xu, D.-P.; Li, H.-B. Impacts of gut bacteria on human health and diseases. Int. J. Mol. Sci. 2015, 16, 7493-7519. [CrossRef]

21. Canfora, E.E.; Meex, R.C.R.; Venema, K.; Blaak, E.E. Gut microbial metabolites in obesity, NAFLD and T2DM. Nat. Rev. Endocrinol. 2019, 15, 261-273. [CrossRef]

22. Kamada, N.; Seo, S.-U.; Chen, G.Y.; Núñez, G. Role of the gut microbiota in immunity and inflammatory disease. Nat. Rev. Immunol. 2013, 13, 321-335. [CrossRef]

23. Araki, Y.; Andoh, A.; Takizawa, J.; Takizawa, W.; Fujiyama, Y. Clostridium butyricum, a probiotic derivative, suppresses dextran sulfate sodium-induced experimental colitis in rats. Int. J. Mol. Med. 2004, 13, 577-580. [CrossRef] [PubMed]

24. Tanaka, M.; Itoh, H. Hypertension as a metabolic disorder and the novel role of the gut. Curr. Hypertens. Rep. 2019, 21, 63. [CrossRef]

25. Liu, J.; Fu, Y.; Zhang, H.; Wang, J.; Zhu, J.; Wang, Y.; Guo, Y.; Wang, G.; Xu, T.; Chu, M.; et al. The hepatoprotective effect of the probiotic Clostridium butyricum against carbon tetrachloride-induced acute liver damage in mice. Food Funct. 2017, 8, 4042-4052. [CrossRef] [PubMed] 
26. Sun, J.; Xu, J.; Yang, B.; Chen, K.; Kong, Y.; Fang, N.; Gong, T.; Wang, F.; Ling, Z.; Liu, J. Effect of Clostridium butyricum against microglia-mediated neuroinflammation in alzheimer's disease via regulating gut microbiota and metabolites butyrate. Mol. Nutr. Food Res. 2020, 64, e1900636. [CrossRef] [PubMed]

27. Sun, J.; Wang, F.; Ling, Z.; Yu, X.; Chen, W.; Li, H.; Jin, J.; Pang, M.; Zhang, H.; Yu, J.; et al. Clostridium butyricum attenuates cerebral ischemia/reperfusion injury in diabetic mice via modulation of gut microbiota. Brain Res. 2016, 1642, 180-188. [CrossRef] [PubMed]

28. Kanai, T.; Mikami, Y.; Hayashi, A. A breakthrough in probiotics: Clostridium butyricum regulates gut homeostasis and antiinflammatory response in inflammatory bowel disease. J. Gastroenterol. 2015, 50, 928-939. [CrossRef]

29. Casula, G.; Cutting, S.M. Probiotics: Spore germination in the gastrointestinal tract. Appl. Environ. Microbiol. 2002, 68, $2344-2352$. [CrossRef] [PubMed]

30. Hachisuka, Y.; Suzuki, I.; Morikawa, K.; Maeda, S. The effect of oxidation-reduction potential on spore germination, outgrowth, and vegetative growth of Clostridium tetani, Clostridium butyricum, and Bacillus subtilis. Microbiol. Immunol. 1982, $26,803-811$. [CrossRef]

31. Yue, Y.; Xu, X.; Yang, B.; Lu, J.; Zhang, S.; Liu, L.; Nassar, K.; Zhang, C.; Zhang, M.; Pang, X.; et al. Stable colonization of orally administered Lactobacillus casei SY13 alters the gut microbiota. Biomed Res. Int. 2020, 2020, 5281639. [CrossRef] [PubMed]

32. Dunne, C.; Murphy, L.; Flynn, S.; O’Mahony, L.; O’Halloran, S.; Feeney, M.; Morrissey, D.; Thornton, G.; Fitzgerald, G.; Daly, C.; et al. Probiotics: From myth to reality. Demonstration of functionality in animal models of disease and in human clinical trials. In Lactic Acid Bacteria: Genetics, Metabolism and Applications, Proceedings of the Sixth Symposium on Lactic Acid Bacteria: Genetics, Metabolism and Applications, Veldhoven, The Netherlands, 19-23 September 1999; Konings, W.N., Kuipers, O.P., In't Veld, J.H.J.H., Eds.; Springer: Dordrecht, The Netherlands, 1999; pp. 279-292.

33. Finnie, I.A.; Dwarakanath, A.D.; Taylor, B.A.; Rhodes, J.M. Colonic mucin synthesis is increased by sodium butyrate. Gut 1995, 36, 93-99. [CrossRef] [PubMed]

34. Sicard, J.-F.; Le Bihan, G.; Vogeleer, P.; Jacques, M.; Harel, J. Interactions of intestinal bacteria with components of the Intestinal mucus. Front. Cell. Infect. Microbiol. 2017, 7, 387. [CrossRef]

35. Tu, Q.V.; McGuckin, M.A.; Mendz, G.L. Campylobacter jejuni response to human mucin muc2: Modulation of colonization and pathogenicity determinants. J. Med. Microbiol. 2008, 57, 795-802. [CrossRef]

36. Rinninella, E.; Raoul, P.; Cintoni, M.; Franceschi, F.; Miggiano, G.A.D.; Gasbarrini, A.; Mele, M.C. What is the healthy gut microbiota composition? a changing ecosystem across age, environment, diet, and diseases. Microorganisms 2019, 7, 14. [CrossRef]

37. Arumugam, M.; Raes, J.; Pelletier, E.; Le Paslier, D.; Yamada, T.; Mende, D.R.; Fernandes, G.R.; Tap, J.; Bruls, T.; Batto, J.M.; et al. Enterotypes of the human gut microbiome. Nature 2011, 473, 174-180. [CrossRef] [PubMed]

38. Magne, F.; Gotteland, M.; Gauthier, L.; Zazueta, A.; Pesoa, S.; Navarrete, P.; Balamurugan, R. The Firmicutes/Bacteroidetes ratio: A relevant marker of gut dysbiosis in obese patients? Nutrients 2020, 12, 1474. [CrossRef] [PubMed]

39. Hou, D.; Zhao, Q.; Yousaf, L.; Xue, Y.; Shen, Q. Whole mung bean (Vigna radiata L.) supplementation prevents high-fat dietinduced obesity and disorders in a lipid profile and modulates gut microbiota in mice. Eur. J. Nutr. 2020, 59, 3617-3634. [CrossRef]

40. Zhang, J.; Song, L.; Wang, Y.; Liu, C.; Zhang, L.; Zhu, S.; Liu, S.; Duan, L. Beneficial effect of butyrate-producing Lachnospiraceae on stress-induced visceral hypersensitivity in rats. J. Gastroen. Hepatol. 2019, 34, 1368-1376. [CrossRef]

41. Qu, W.; Yuan, X.; Zhao, J.; Zhang, Y.; Hu, J.; Wang, J.; Li, J. Dietary advanced glycation end products modify gut microbial composition and partially increase colon permeability in rats. Mol. Nutr. Food Res. 2017, 61, 1700118. [CrossRef]

42. Hu, S.; Wang, J.; Xu, Y.; Yang, H.; Wang, J.; Xue, C.; Yan, X.; Su, L. Anti-inflammation effects of fucosylated chondroitin sulphate from acaudina molpadioides by altering gut microbiota in obese mice. Food Funct. 2019, 10, 1736-1746. [CrossRef] [PubMed]

43. Cui, H.-X.; Hu, Y.-N.; Li, J.-W.; Yuan, K. Hypoglycemic mechanism of the berberine organic acid salt under the synergistic effect of intestinal flora and oxidative stress. Oxid. Med. Cell. Longev. 2018, 2018, 8930374. [CrossRef] [PubMed]

44. Ruppin, H.; Bar-Meir, S.; Soergel, K.H.; Wood, C.M.; Schmitt, M.G., Jr. Absorption of short-chain fatty acids by the colon. Gastroenterology 1980, 78, 1500-1507. [CrossRef]

45. Rechkemmer, G.; Rönnau, K.; von Engelhardt, W. Fermentation of polysaccharides and absorption of short chain fatty acids in the mammalian hindgut. Comp. Biochem. Phys. A 1988, 90, 563-568. [CrossRef]

46. Kasubuchi, M.; Hasegawa, S.; Hiramatsu, T.; Ichimura, A.; Kimura, I. Dietary gut microbial metabolites, short-chain fatty acids, and host metabolic regulation. Nutrients 2015, 7, 2839-2849. [CrossRef] 